\title{
The many faces of social anxiety disorder
}

\author{
Hans-Ulrich Wittchen \\ Institute of Clinical Psychology and Psychotherapy, Technical University of Dresden, and \\ Max Planck institute of Psychiatry, Munich, Germany
}

Correspondence to Hans-Ulrich Wittchen, Max Planck Institute of Psychiatry, Clinical Psychology and Epidemiology, Kraepelinstrasse 10, Munich, Germany. Tel: +498930622 546; fax: +49 89 30622 544; e-mail: wittchen@mpipsykl.mpg.de

\begin{abstract}
Social anxiety disorder, also known as social phobia, is one of the most prevalent anxiety disorders, affecting $7-13 \%$ of subjects in the community at some time in their lives. Despite being eminently treatable, it remains largely under-recognised and, therefore, undertreated. The disorder is characterized by a fear of scrutiny by others, with sufferers experiencing excessive anxiety in social and performance situations. This excessive anxiety usually leads to avoidance behaviour that can severely affect normal daily living. With onset commonly occurring during childhood or adolescence, social anxiety disorder may disrupt normal patterns of development of social and personal relationships, often having a long-term impact on emotional stability in social or working life. If left untreated, the course of social anxiety disorder is frequently complicated with comorbid conditions, particularly major depression or substance abuse. This review assesses the size of the clinical problem by evaluating current and lifetime prevalence estimates, age of onset, risk factors and evolution of the clinical course; thereby providing the rationale for early recognition and prompt treatment. Int Clin Psychopharmacol 15 (suppl 1):\$7-S12 02000 Lippincott Williams \& Wilkins
\end{abstract}

Keywords: social anxiety disorder, prevalence, onset, family genetic factors, behavioural inhibition, comorbidity

\section{INTRODUCTION}

Anxiety in stressful social situations is, generally speaking, a normal and even ubiquitous human response to challenging situations and a certain level of distress and anxiety is even necessary for individuals to develop and to function normally. However, such anxiety reactions, in terms of their frequency and the degree of anxiety symptoms related to it, may become exaggerated to the point of interfering with normal functioning. Social anxiety disorder (social phobia) is characterized by persistent, unreasonably strong, frequently overwhelming fears of social and performance situations where individuals are fearful of being scrutinized by other people and being subject to negative evaluation.

In social anxiety disorder, the fear can occur in virtually all types of everyday situations involving social interactions, such as speaking with others or attending a meeting or a party, and also in special situations, such as taking a test or examination and public speaking. In some sufferers, the disorder can be confined to a small circumscribed set of situations. In others, it can occur in a wide variety of social situations; in these cases, a diagnosis of 'generalized social anxiety disorder' is assigned. When anticipating or experiencing exposure to a provoking situation, the sufferer will almost invariably have an anxiety reaction, which may take the form of a panic attack. The distress caused by the anxiety almost always leads to pronounced and persistent avoidance of the situation; this sense of distress and the subsequent avoidant behaviour usually significantly limits the individual's social development, for example with regard to social relationships, thereby restricting social life and achievements in both education and at work.

This review examines some of the more recent evidence focusing on descriptive and causal analytic epidemiological studies of social anxiety disorder, in terms of its prevalence, clinical evolution and associated complications. Additionally, the burden of the disorder on medical services is assessed to demonstrate how early recognition and treatment could both reduce healthcare utilization and minimize the individual's suffering. 


\section{PREVALENCE}

In the past 10 years, numerous studies have investigated the frequency of strictly-defined social anxiety disorder in various parts of the world by means of large-scale representative samples of the general population (Kessler et al., 1994; Lin et al., 1996; Wittchen et al., 1998; Lecrubier et al., 2000; Heimberg et al., 2000) and, less frequently, in primary care (Weiller et al., 1996). Whether using DSM-IV or DSM-III-R criteria, the 1-year and lifetime prevalence rates of this condition have demonstrated it to be a common anxiety disorder in all populations assessed. In the United States, for example, the National Comorbidity Survey (NCS) (Kessler et al., 1994) evaluated the 1-year and lifetime prevalences as 8 and $13 \%$, respectively; in Canada, the Ontario Health Survey (Lin et al., 1996) reported 7 and 13\%, respectively; and in Europe, the Early Developmental Stages of Psychopathology Study (EDSP) (Wittchen et al., 1998) in 14- to 24-year-olds reported 5 and $10 \%$, respectively. In primary-care offices, Weiller et al. (1996) reported a current prevalence estimate of 5-7\% and, as part of this international WHO study, further highlighted that while this disorder is common, it is rarely correctly diagnosed at the primary care level; in the main, patients were diagnosed as psychological cases, but not necessarily as suffering from social anxiety disorder.

Few data are yet available about the prevalence of the generalized subtype of social anxiety disorder, which is considered to be a more severe form seen frequently in specialist settings according to most clinical studies (Heimberg et al., 1990; Mannuzza et al., 1995; Stein et al, 1998). In the EDSP study (Wittchen et al., 1998), longitudinal data showed that although both subtypes had a comparable current prevalence (3.6 nongeneralized versus 3.0 generalized), the cumulative lifetime likelihood of experiencing nongeneralized social anxiety disorder was noticeably higher (6.4 nongeneralized versus 3.9 generalized). Data from this study support the view that there is a greater persistence of the more severe, generalized form of social anxiety disorder, while the nongeneralized subtype remits spontaneously more frequently (Müller, 2000).

\section{ONSET}

Unlike agoraphobia, panic disorder, generalized anxiety disorder, and depressive disorder, social anxiety disorder almost always has an early onset, in childhood or adolescence (Beidel, 1998). Consistently across all epidemiological studies only a few cases have been identified that reported an onset of the condition after the age of 21 years. Even in those with a later onset of the full disorder, in most cases significant signs of the dis- order have been evident in early life. Given the early onset of social anxiety disorder, it is noteworthy from a differential diagnostic perspective that according to DSM-IV stipulations, it is not recommended to summarize the disorder under the 'avoidant type' of personality disorders. As social anxiety disorder is a more specific condition for which established treatment exists, and given that personality disorder should not include significant clinical syndromes of the axis I disorders, preference should be given to the diagnosis of social anxiety disorder rather than to a personality disorder.

From an aetiological perspective, the early onset of social anxiety disorder points to the significance of potential genetic and familial links as well as temperamental variations as significant contributors to the onset of this condition. Furthermore, it should be highlighted that although phobic disorders during childhood and adolescence are frequently a transient developmental phenomenon, (e.g. in the case of animal, darkness and separation phobias that occur at certain ages more or less clearly in the development of the majority of children for some time but then spontaneously remit at least in early puberty), the fear of social interaction is a comparatively more stable phenomenon (Achenbach, 1985; Beidel, 1998) and is more likely to persist both in childhood and during adolescence into adulthood.

\section{RISK FACTORS}

There are numerous and quite unique factors that have been associated with an increased risk for first onset of social anxiety disorder and its persistence, both from clinical as well as epidemiological studies (Wittchen et al., 1999a; Lieb et al., 2000; Magee et al., 1996). Familial and developmental factors are of special importance, in that they exist before the onset of the disorder, and thus might be most informative in terms of the underlying vulnerabilities.

Several studies have demonstrated that social anxiety disorder runs in families, with both familial genetic and environmental contributions. Twin studies in females suggest concordance of $24.4 \%$ between monozygotic twins and $15.3 \%$ between dizygotic twins. In addition to twin studies, other types of family studies also provide support for familial aggregations (Beidel, 1998; Fyer, 1993). These studies were performed in patient samples, but further evidence was recently provided in a study of a community sample of 1053 parents (Lieb et al., 2000) that prospectively examined the 5 -year risk of their offspring developing social anxiety disorder during adolescence. Three groups of parents were identified: (1) parents with no lifetime diagnosis of a mental disorder; (2) parents with a lifetime 
diagnosis of a mental disorder, for example, depressive disorder, but not social anxiety disorder; (3) those who fulfilled the diagnostic criteria of social anxiety disorder. The children of the parents in the last group (3) were found to be much more likely to develop social anxiety disorder during adolescence, with an odds ratio (OR) of 4.7 (95\% CI: 1.7-13.0). This study also confirmed that, independent from this parental association, family environmental factors, specifically parental overprotection (OR: 1.4) and rejection (OR: 1.4), were additional risk factors for the onset of social anxiety disorder, underlining significant links between familial genetic and environmental factors.

Among other early antecedents of social anxiety disorder, behavioural inhibition (BI) was repeatedly confirmed as a strong contributory behaviouraltemperamental risk factor (Rosenbaum et al., 1993; Kagan, 1997; Beidel and Turner, 1997). BI refers to neurobiological and psychological constructs that describe the fear of the unfamiliar, this is defined as a tendency for children to react consistently to unfamiliar events with restrained withdrawal and shyness. Assessable even in young children by means of parent reports and behavioural child observation, BI has been repeatedly shown to provide highly significant associations with the development of social anxiety disorder in later life. Measuring these temperamental states in children shows that those with higher BI values are significantly more likely to develop social anxiety disorder than others (Kagan et al., 1988; Hayward et al., 1998; Beidel, 1998; Lieb et al., 2000). Additionally, as high BI values in children have been associated with high $B I$ values in parents, we can assume that temperament is a risk factor for the development of social anxiety disorder. Müller (2000) recently confirmed this, in a large representative community sample of 3021 adolescents and young adults (OR: 4.5); however, whether this temperamental disposition is a necessary or sufficient aetiological factor remains to be determined (Beidel and Turner, 1997). Furthermore, significant risk factors also suggested include trauma, separation events, and preceding phobias (Stemberger et al., 1995; Müller, 2000). With regard to sociodemographic factors that may also influence the onset of social anxiety disorder, it is so far not known to what degree these factors are consequences or correlates of the disorder. Poor financial situation (OR: 1.7), low social class (OR: 3.1), never being married (OR: 2.1 ), unemployment (OR: 2.3), and poor education (OR: 3.1 ) frequently relate to lack of socialization during the early stages of the disorder. In addition, factors such as age below 21 years (OR: 6.1), and female sex (OR: 1.9) are direct correlates of social anxiety disorder (Magee et al., 1996; Müller, 2000).

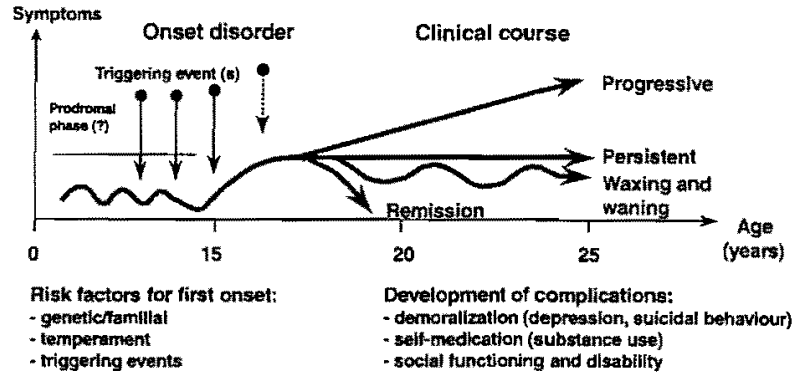

Figure 1. Evolution of social anxiety disorder.

\section{Evolution, clinical course and comorbidity}

Beyond numerous in-depth clinical studies of smaller samples, the evolution of social anxiety disorder has recently been followed by the EDSP study of 3021 community cases (Wittchen et al., 1998). The majority of cases of social anxiety disorder have emerged by the time an individual has reached their early $20 \mathrm{~s}$, when either a progressive worsening or a persistent course will have been established. Many individuals, especially in late adolescence, will also report a waxing and waning course (Wittchen et al., 1999a), whereby social problems can be temporarily ameliorated by virtue of their attachment to a particular partner in a calming relationship, but then a new challenge in life will precipitate the re-presentation of the full disorder (Müller, 2000) (Fig. 1). The main outcome of the EDSP study suggests that irrespective of some variations in severity (fluctuation between symptomatic, subthreshold and threshold stages), considerably fewer individuals with social anxiety disorder will have a stable and spontaneous remission; with the exception of specific childhood phobias, the remission rate in social anxiety disorder is the lowest amongst all anxiety disorders (Wittchen et al., 1999a). It is noteworthy however, that the EDSP study was also unable to identify what specific events actually provoke the onset of the disorder (see Fig. 1). Behavioural theories of aetiologies suggest three potential mechanisms: direct conditioning, in which fear develops from exposure to a critical or traumatic event; vicarious learning (observation signals in a traumatic situation); and information transfer, by the verbal or non-verbal transmission of information about social situations. Reasons both for and against these perspectives are possible, however as in earlier studies, the retrospective nature of the assessments prevents firm conclusions from being drawn.

The evolutionary model described above suggests that in certain individuals there is a vulnerability, such that when confronted with a challenging or critical situation they are more likely to develop enduring social fear and subsequent avoidance. This can lead to a 
restriction in their lifestyle, and prevent them from making the most of available opportunities. These individuals can develop disabilities in their school, work, and social life, with particular problems in the initiation and maintenance of relationships with the opposite sex. Sufferers may experience demoralization and even full blown depressive disorder, and develop inadequate coping behaviours; for example, using alcohol or nicotine in order to alleviate their suffering. Over time, increased disability and a reduced quality of life could be expected in such individuals, as well as increasing rates of comorbidity with secondary mental disorders.

Some of these hypotheses have been examined in several studies, one of which was a retrospective analysis of the NCS (Kessler et al., 1999). The findings demonstrated a high prevalence of comorbidity, with many individuals suffering from social anxiety disorder also meeting the diagnostic criteria for major depression or dysthymia. Specifically, it showed that the probability for a comorbid mental disorder was approximately $50 \%$. With regard to the course of comorbidity, social anxiety disorder was generally the primary condition with major depression and dysthymia being secondary disorders.

There are recognized limitations of a retrospective analysis. However, a more recent prospective study demonstrates similar findings (EDSP data: Wittchen et al., 1999a). In this study, adolescents and young adults with social anxiety disorder, of the generalized or nongeneralized subtype, but without any significant depressive symptoms, were assessed over a period of several years, and the prospective occurrence of depressive episodes was monitored. It was found that individuals with social anxiety disorder were 3.5 times more likely to develop a major depressive episode than those with no social anxiety disorder; furthermore, the risk for secondary depressive disorders was slightly higher in generalized as opposed to nongeneralized social anxiety disorder. It was noteworthy that subjects with social anxiety disorder already comorbid with depression were at a further and greatly increased risk of either having no remission of depression, or experiencing a new episode of depression (OR: 8.7). This suggests that social anxiety disorder is not only a risk factor for depression, but is also associated with a more malignant course and character of subsequent depressive illness (Fig. 2).

The EDSP survey also examined the probability that individuals with social anxiety disorder have an increased risk for developing substance abuse disorder. This was verified, and it was found that sufferers have an increased intake of, and dependence on, legal substances such as alcohol (OR: 1.8) (Fig. 2). The

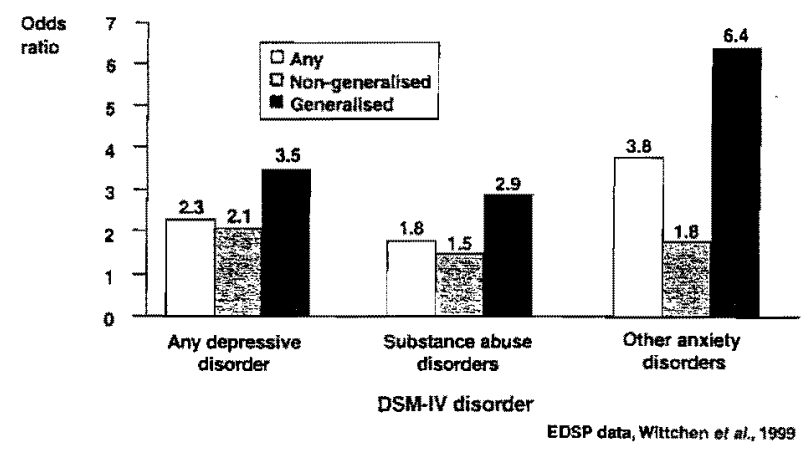

Figure 2. Risk of developing comorbid disorders.

individuals were also more likely to develop nicotine dependence (Sonntag et al., 2000). Abuse of, or dependence on, illegal drugs was less likely to occur, probably due to the subjects being anxious about breaking the law. Individuals with social anxiety disorder also manifested symptoms associated with other anxiety disorders and in some cases the symptomatology was sufficient to meet the diagnostic criteria for another anxiety disorder (Fig. 2).

Demoralization as a result of enduring social anxiety disorder can precipitate suicidal ideation and may lead to suicide attempts. This was investigated in a community sample, where individuals with social anxiety disorder, both in its pure form and in the comorbid condition, were compared with controls (Wunderlich et al., 1998). It was found that, although subjects with the pure form were much more likely to 'feel like wanting to die' and 'felt so low that they had suicidal ideation', the rate of suicide attempts did not differ from that in the control group. In individuals with comorbid depression however, the risk of suicide attempts was greatly increased (Table 1). Thus the comorbid picture of social anxiety disorder plus major depression represents a particularly high risk group for suicidal ideation and suicide attempts.

In summary, patients with social anxiety disorder are at an increased risk of developing secondary depressive episodes, and social anxiety disorder can be considered a risk factor for depression. Furthermore, the depression is likely to develop at an earlier age than in a matched control group, and secondary depression is more persistent than a pure depressive disorder. These patients are also more likely to develop substance abuse and dependence.

\section{Disability and quality of life}

Functional disability and quality of life assessment has been used as a more generic measure of individual suf- 
Table 1. Sulcidal behaviour in social anxiety disorder (Wunderlich et al., 1998)

\begin{tabular}{lccc}
\hline & $\begin{array}{c}\text { Controls } \\
(\%)\end{array}$ & $\begin{array}{c}\text { Pure } \\
(\%)\end{array}$ & $\begin{array}{c}\text { Comorbid } \\
(\%)\end{array}$ \\
\hline Suicide attempts & 0.2 & 0.9 & 15.7 \\
Thought a lot about death & 11.3 & 26.8 & 53.8 \\
Felt like you wanted to die & 4.2 & 8.9 & 27.7 \\
Felt so low you wanted to & 2.1 & 9.8 & 37.3 \\
commit suicide & & & \\
\hline
\end{tabular}

fering (Davidson et al., 1994; Magee et al., 1996). The degree of functional impairment in various social roles (e.g. days unable to work, reduced work performance and impaired social interaction) has been assessed using the Liebowitz Social Disability Scale (LSDS) or the CIDI Disability Module (CDM), and the extent to which an individual's health impacts on their day-today functioning and enjoyment of life has been assessed using the Medical Outcomes Study Short Form 36 (SF36), Quality Of Life Inventory (QOLI) and the Illness Intrusiveness Ratings Scale (IIRS).

Using a control group comprised of individuals with another socially-embarrassing disorder, herpes infection, reduction in work productivity was measured using the Work Productivity and Activity Index (WPAI) score (Wittchen et al., 1999b). When compared with the controls $(1.5 \%)$, a significantly greater reduction in work productivity $(P<0.001)$ was demonstrated by both the individuals with pure social anxiety disorder $(11.4 \%)$ and those with comorbid depressive symptoms (12.4\%).

Assessing quality of life, using SF-36, both pure and comorbid patients were compared with the control group and with depressed outpatients (Wittchen and Beloch, 1996; Wittchen et al., 1999b). Patients with comorbid social anxiety disorder exhibited quality of life impairment similar to depressed outpatients (markedly, $31.4 \%$ vs. $28.7 \%$; severely, $51.0 \%$ vs. $31.4 \%$ ). However, a surprising finding was that a similar pattern also occurred in those patients with pure social anxiety disorder ( 24.6 and $23.1 \%$, respectively).

More detailed analyses of quality of life impairment have demonstrated an increased risk of leaving school early (OR: 1.72), disability in major social roles (OR: 4.15) and marked dissatisfaction with friends (OR: 5.19), leisure activities (OR: 2.37) and income (OR: 1.87) (Stein et al., 1999). Additionally, long-term findings have shown that social anxiety disorder is associated with increased likelihood of being unem- ployed (OR: 2.8), lower educational attainment in terms of university and professional training (OR: 4.2) and increased probability of being single or divorced (OR: 2.8) (Wittchen et al., 1999a).

The impact of this reduced quality of life and increased functional disability on healthcare utilization was assessed by the Dean Foundation Study (D. J. Katzelnick, presented at meeting of the American Psychiatric Association, 1999, New Orleans, USA). Using a large managed care population of 10000 people, the study found that current costs associated with social anxiety disorder, compared with matched controls, represented a significantly higher $(P=0.04)$ health-economic burden (approximately $\$ 2400$ versus approximately $\$ 1900$ ).

Usage of medical services has remained remarkably stable over the years, with utilization in the 1980 s being similar to that reported in 1992 (Schneier et al., 1992). Pure social anxiety disorder does not incur high utilization rates per se and patients tend to receive little psychiatric or general outpatient treatment, unless the social anxiety disorder becomes complicated by another disorder.

\section{SUMMARY AND CONCLUSIONS}

Social anxiety disorder is a common condition, with current prevalence estimates in the range of $5-7 \%$ and a lifetime risk of $7-13 \%$. It has an early onset and, without appropriate intervention, it has a disproportionately higher risk of persistence, compared with other anxiety disorders. Presentation differs between age groups, the disorder in teenagers and in those in their early $20 \mathrm{~s}$ tending to look different in terms of types of problem and the associated distress, to that expected in the 30s and 40 s age groups, when the individual has already endured 20 years of suffering and disability. There is an increased risk of depression and substance abuse disorders even in adolescence, in addition to an increased risk of psychosocial impairment and disability resembling that experienced by depressed outpatients. This is particularly true in cases affected by generalized social anxiety disorder, which might have slightly different aetiological pathways than the nongeneralized type.

Individuals who develop comorbid conditions have a more severe level of disability. Early recognition, diagnosis and treatment of social anxiety disorder could minimize sufferers' problems throughout their subsequent lives, preventing the development of comorbidity and a worsened prognosis. Developing models for early recognition and treatment should improve outcome for the patient, as well as reducing future demand on healthcare resources. 


\section{REFERENCES}

Achenbach TM (1985). Assessment of anxiety in children. In: Anxiety and the anxiety disorders Tuma AH, Maser JD (editors). Hillsdale, NJ: Lawrence Erlbaum Associates.

Beidel DC (1998). Social anxiety disorder: etiology and early clínical presentation. J Clin Psychiatry 59 (suppl 17):2731.

Beidel DC, Turner SM (1997). At risk for anxiety: I. Psychopathology in the offspring of anxious parents. $J$ Am Acad Child Adolesc Psychiatry 36:918-924.

Davidson JR, Hughes DC, George LK, Blazer DG (1994). The boundary of social phobia. Exploring the threshold. Arch Gen Psychiatry 51:975-983.

Fyer AJ (1993). Heritability of social anxiety: a brief review. $J$ Clin Psychiatry 54 (suppl 12):10-12.

Hayward C, Killen JD, Kraemer HC, Taylor CB (1998). Linking self-reported childhood behavioral inhibition to adolescent social phobia. J Am Acad Child Adolesc Psychiatry 37:1308-1316.

Heimberg RG, Hope DA, Dodge CS, Becker RE (1990). DSM-III-R subtypes of social phobia. Comparison of generalized social phobias anad public speaking phobics. $J$ Nerv Mental Dis 178:172-179.

Heimberg RG, Stein MB, Hiripi E, Kessler RC (2000). Trends in the prevalence of social phobia in the United States: a synthetic cohort analysis of changes over four decades. Eur Psychiatry 15:29-37.

Kagan J (1997). Temperament and the reactions to unfamiliarity. Child Dev 68:139-43.

Kagan J, Reznick J, Snidman N (1988). Temperamental influences on reactions to unfamiliarity and challenge. $A d v$ Exp Med Biol 245;319-339.

Kessler RC, McGonagle KA, Zhao S, Nelson CB, Hughes $M$, Eshleman S et al. (1994). Lifetime and 12-month prevalence of DSM-III-R psychiatric disorders in the United States: results from the National Comorbidity Survey. Arch Gen Psychiatry 51:8-19.

Kessler RC, Stang P, Wittchen H-U, Stein M, Walters EE (1999). Lifetime co-morbidities between social phobia and mood disorders in the US National Comorbidity Survey. Psychol Med 29:555-567.

Lecrubier Y, Wittchen HU, Faravelli C, Bobes J, Patel A, Knapp M (2000). A European perspective on social anxiety disorder. Eur Psychiatry 15:5-16.

Lieb R, Wittchen H-U, Höfler M, Fuetsch M, Stein M, Merikangas KR (2000). Does parental psychopathology increase the risk for social phobia in offspring? Results from a longitudinal epidemiological study in adolescents and their parents. Arch Gen Psychiatry (in press).

Lin E, Goering P, Offord DR, Campbell D, Boyle MH (1996). The use of mental health services in Ontario: epidemiologic findings. Can J Psychiatry 41:572-577.
Magee WJ, Eaton WW, Wittchen H-U, McGonagle KA, Kessler RC (1996). Agoraphobia, simple phobia, and social phobia in the National Comorbidity Survey. Arch Gen Psychiatry 53:159-168.

Mannuzza S, Schneier FR, Chapman TF, Liebowitz MR, Klein DF, Fyer AJ (1995). Generalized social phobia. Reliability and validity. Arch Gen Psychiatry 52:230-237.

Müller N (2000). Frühstadien der sozialen phobie: risiken, erscheinungsformen und konsequenzen. Dissertation. University of Bamberg (in press).

Rosenbaum JF, Biederman J, Bolduc-Murphy EA, Faraone SV, Chaloff J, Hirschfeld DR et al. (1993). Behavioral inhibition in childhood: a risk factor for anxiety disorders. Harv Rev Psychiatry 1:2-16.

Schneier FR, Johnson J, Hornig CD, Liebowitz MR, Weissman KM (1992). Social phobia. Comorbidity and morbidity in an epidemiologic sample. Arch Gen Psychia. try 49: 282-288.

Sonntag H, Wittchen HU, Höfler M, Kessler RC, Stein HB (2000). Are social fears and DSM-IV social anxiety disorder associated with smoking and nicotine dependence in adolescents and young adults? Eur Psychiatry 15:6774.

Stein MB, Chartier MJ, Hazen AL, Kozak MV, Tancer ME, Lander $S$ et al. (1998). A direct-interview family study of generalized social phobia. Am J Psychiatry 155:90-97.

Stein MB, McQuaid JR, Laffaye C, McCahill ME (1999). Social phobia in the primary care medical setting. $J$ Fam Pract 48:514-519.

Stemberger RT, Turner SM, Beidel DC, Calhoun KS (1995). Social phobia: an analysis of possible developmental factors. J Abnorm Psychol 104: 526-531.

Weiller E, Bisserbe JC, Boyer P, Lepine JP, Lecrubier Y (1996). Social phobia in general health care: an unrecognized undertreated disabling disorder. $B r J$ Psychiatry 168:169-174.

Wittchen H-U, Beloch E (1996). The impact of social phobia on quality of life. Int Clin Psychopharmacol 11 (suppl 3): $15-23$.

Wittchen H-U, Nelson CB, Lachner G (1998). Prevalence of mental disorders and psychosocial impairments in adolescents and young adults. Psychol Med 28:109-126.

Wittchen H-U, Nelson CB, Kessler RC (1999a). Social fears and DSM-IV social phobia in a community sample of adolescents and young adults: prevalence, risk factors and comorbidity. Psychol Med 29:309-329.

Wittchen H-U, Fuetsch M, Sonntag H, Müller N, Liebowitz $\mathrm{M}(1999 \mathrm{~b})$. Disability and quality of life in pure and comorbid social phobia: findings from a controlled study. Eur Psychiatry 14:118-131.

Wunderlich U, Bronisch T, Wittchen H-U (1998). Comorbidity patterns in adolescents and young adults with suicide attempts. Eur Arch Psychiatry Clin Neurosci 248: $87-95$. 